\title{
A systematic review of endovascular stent-electrode arrays, a minimally invasive approach to brain-machine interfaces
}

\author{
Sauson Soldozy, BA, ${ }^{1}$ Steven Young, BA, ${ }^{1}$ Jeyan S. Kumar, MD, ${ }^{1}$ Stepan Capek, MD, ${ }^{1}$ \\ Daniel R. Felbaum, MD, ${ }^{2}$ Walter C. Jean, MD, ${ }^{3}$ Min S. Park, MD, ${ }^{1}$ and Hasan R. Syed, MD ${ }^{1}$ \\ 1Department of Neurological Surgery, University of Virginia Health System, Charlottesville, Virginia; ${ }^{2 D e p a r t m e n t ~ o f ~}$ \\ Neurosurgery, Georgetown University, Washington, DC; and '3epartment of Neurosurgery, George Washington University, \\ Washington, DC
}

OBJECTIVE The goal of this study was to systematically review the feasibility and safety of minimally invasive neurovascular approaches to brain-machine interfaces (BMIs).

METHODS A systematic literature review was performed using the PubMed database for studies published between 1986 and 2019. All studies assessing endovascular neural interfaces were included. Additional studies were selected based on review of references of selected articles and review articles.

RESULTS Of the 53 total articles identified in the original literature search, 12 studies were ultimately selected. An additional 10 articles were included from other sources, resulting in a total of 22 studies included in this systematic review. This includes primarily preclinical studies comparing endovascular electrode recordings with subdural and epidural electrodes, as well as studies evaluating stent-electrode gauge and material type. In addition, several clinical studies are also included.

CONCLUSIONS Endovascular stent-electrode arrays provide a minimally invasive approach to BMls. Stent-electrode placement has been shown to be both efficacious and safe, although further data are necessary to draw comparisons between subdural and epidural electrode measurements given the heterogeneity of the studies included. Greater access to deep-seated brain regions is now more feasible with stent-electrode arrays; however, further validation is needed in large clinical trials to optimize this neural interface. This includes the determination of ideal electrode material type, venous versus arterial approaches, the feasibility of deep brain stimulation, and more streamlined computational decoding techniques.

https://thejns.org/doi/abs/10.3171/2020.4.FOCUS20186

KEYWORDS brain-machine interface; endovascular stent-electrode; minimally invasive; neural interface

$\mathrm{B}$ RAIN-MACHINE interface (BMI) refers to the direct communication between the brain and external hardware capable of measuring and interpreting neuronal signaling. Assisting patients with neurorehabilitation, BMI technology enables translation of neuronal activity into control signals for assistive devices such as wheelchairs and robotic prosthetics. ${ }^{1}$ While an electrocorticographic brain interface has proven efficacious, traditional electrode placement imparts risks such as hematoma development and sequelae of chronic blood-brain barrier breach. ${ }^{2,3}$ Moreover, direct electrode array implantation is poorly suited for measurement of signals in deep cortical and brain structures. ${ }^{4}$ For this reason, less invasive endovascular approaches for electrode placement have been explored.

First shown to be technically feasible in humans nearly 4 decades ago, intracranial intravascular EEG represents a novel approach to signal detection..$^{5-7}$ Additionally, interpretation and decoding of neural data has also been demonstrated, suggesting signals recorded with a stentelectrode array (Stentrode, Synchron) are able to be classified into the appropriate motor movements. ${ }^{8}$ Furthermore,

ABBREVIATIONS BMI = brain-machine interface; DBS = deep brain stimulation; SNR = signal-to-noise ratio; $S S S$ = superior sagittal sinus; SVM = support vector machine. SUBMITTED March 2, 2020. ACCEPTED April 20, 2020. INCLUDE WHEN CITING DOI: 10.3171/2020.4.FOCUS20186. 


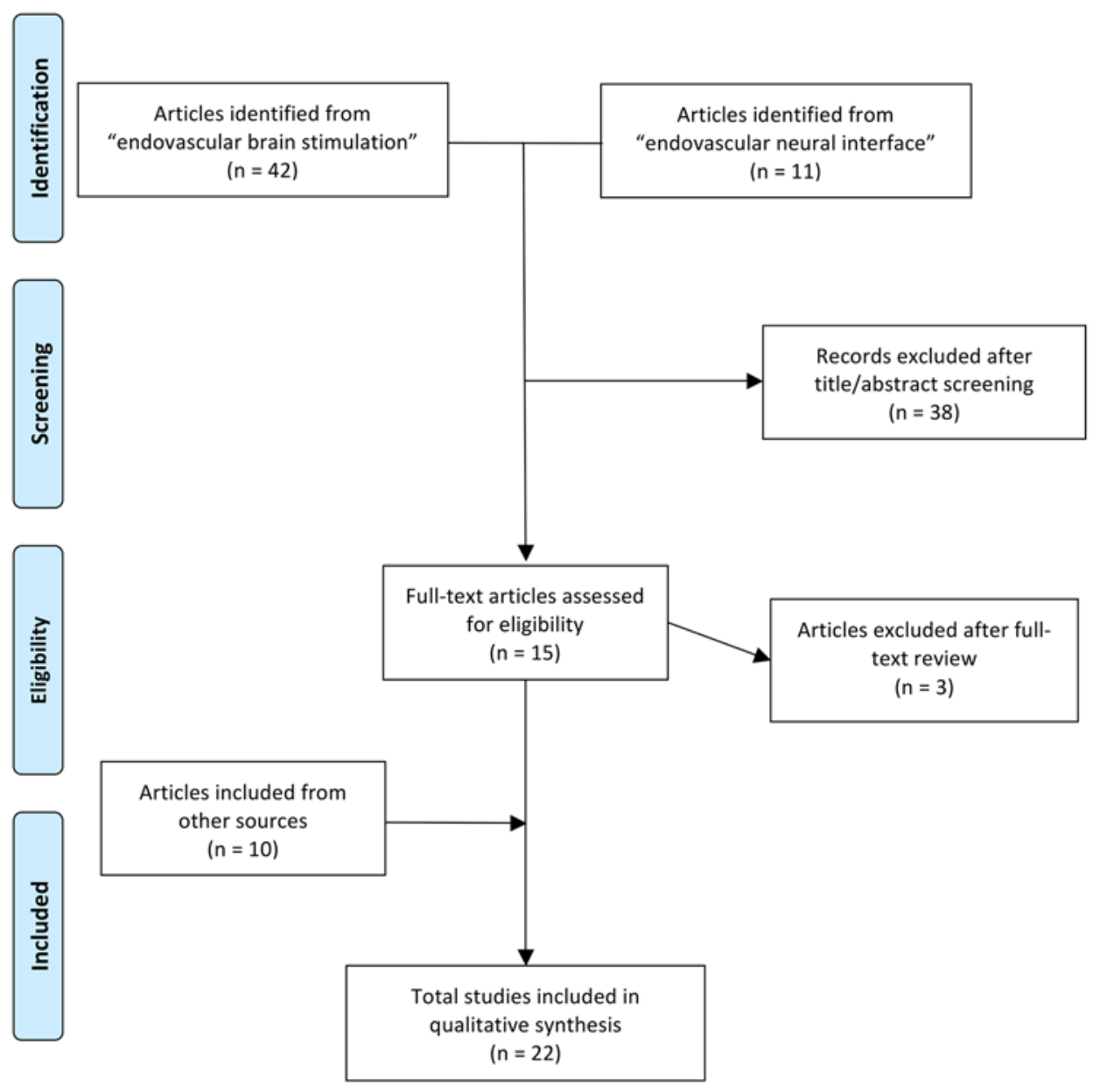

FIG. 1. Diagram of study selection process.

access to sulci and deep brain structures is another potential advantage of an endovascular approach. ${ }^{9}$

In this systematic review, the authors provide an overview of endovascular stent-electrode arrays, including a discussion of safety and efficacy with respect to signal acquisition and decoding, brain stimulation, and electrode characteristics such as material and placement duration.

\section{Methods}

A literature search using PubMed and MEDLINE databases was performed with the search terms "endovascular brain stimulation" and "endovascular neural interface," yielding 53 articles. After reviewing the titles and abstracts for relevance, 15 articles were selected for fulltext review, and 12 studies were selected. An additional 10 studies were included after reviewing the sources of included articles and review articles, for a total of 22 studies. ${ }^{9-11}$ Data from the included studies were independently reviewed and extracted by the first and second authors. A PRISMA flow diagram detailing methods for selection of studies is presented in Fig. 1. Review articles, abstracts, editorials without original data, and articles not written in English were excluded.

\section{Results}

A total of 22 articles were included and are summarized in Table 1. This includes articles assessing the feasibility and efficacy of an endovascular BMI in primarily ovine models, including comparisons of signal detection to traditional means as well as an assessment of chronic stent-electrode placement and stent material. The findings of these studies are summarized below.

\section{Endovascular Signal Detection and Decoding}

John et al. ${ }^{6}$ explored the potential distortive effects of the dura mater and blood vessels on recording signal quality by comparing endovascular electrode arrays with conventional subdural and epidural interfaces. Electrodes of various sizes and locations were tested with respect to bandwidth, sensitivity, signal-to-noise ratio (SNR), spatial profile, and ability to decode the recordings. All measurements were taken 3-4 weeks following electrode placement into sheep to allow adequate time for the arrays to be integrated into the vessels.

Regarding bandwidth, there was no significant effect caused by array location, electrode size, recording location, or size of electrode. With respect to SNR, there was 


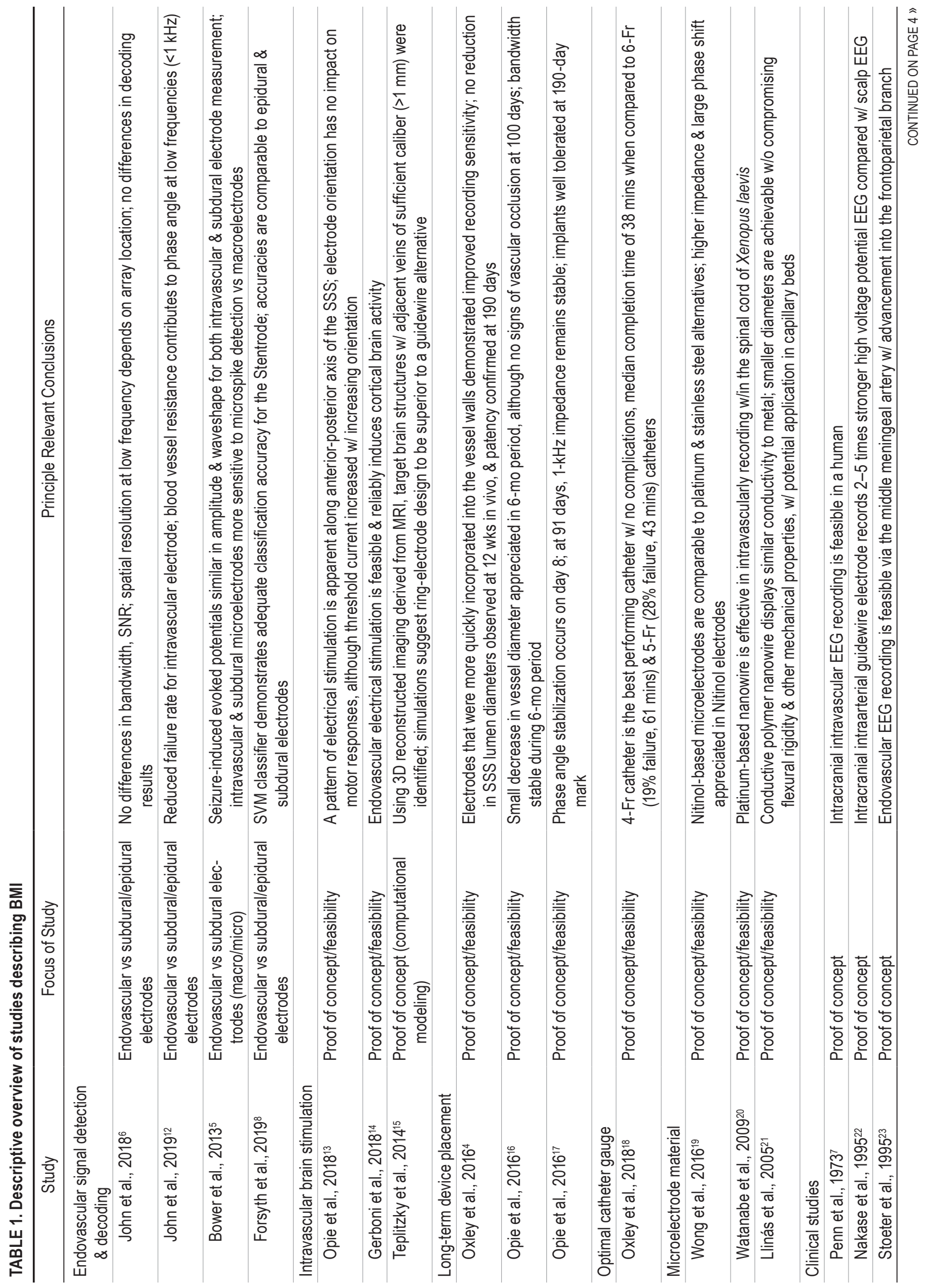


no significant effect of size or location. However, spatial resolution was dependent on array location, varying significantly only in the 8 - to $24-\mathrm{Hz}$ frequency band $\left(\chi^{2}=\right.$ $11.42, \mathrm{p}=0.003)$. Decoding results indicated there was no appreciable difference in performance between the epidural, subdural, and endovascular arrays.

In another study, John et al. ${ }^{12}$ utilized electrical impedance spectroscopy to quantify the electrochemical properties for subdural, intravascular, and epidural arrays. The intravascular arrays were implanted in the superior sagittal sinus (SSS) adjacent to the motor cortex of 5 adult sheep, while subdural and epidural arrays were implanted on either hemisphere. At the time of testing, all animals had some degree of electrode failure, with a failure rate of $40 \%-60 \%$ for intravascular electrodes compared to $33 \%-73 \%$ in the subdural and epidural arrays.

The authors demonstrated that the electrical properties of intravascular arrays were impacted at lower frequency ranges. Specifically, the intravascular arrays demonstrated higher phase angles at frequencies less than $1 \mathrm{kHz}$ when compared to subdural and epidural arrays. This suggests a sensitivity to the blood vessel endothelium at this frequency range, and a greater resistance is imparted by the blood vessel-electrode interface. At higher frequencies, though, the dura and CSF are thought to impact phase response and impedance magnitude. While the dura is thin and resistive, the high conductivity of the CSF is believed to have a larger impact on the electrical properties of the electrode-tissue interface than dura.

Bower et al..$^{5}$ explored the potential of continuous endovascular electrophysiological monitoring as a recording device for epileptic activity, comparing endovascular and subdural electrode arrays in pigs. Macro- and microelectrode-containing catheters were placed in the SSS parallel with a subdural electrode array. Seizures were induced via direct cortical injection of penicillin. The epileptiform spikes recorded by the intravascular device were similar in amplitude and waveshapes to those recorded by the subdural interface. However, intravenous recordings detected high-frequency signals that were missed by surrounding clinical macroelectrodes. These "microspikes" are thought to represent seizure activity within a spatially restricted neural population, too small to be detected by clinical macroelectrodes. Subdural microelectrodes also picked up these microspikes, suggesting vascular artifact to be unlikely in this scenario. It should be noted that frequency response curves demonstrated a reduction in signal detection by $11 \%$ and $24.2 \%$ at $30 \mathrm{~Hz}$ and $100 \mathrm{~Hz}$, respectively, among the intravascular electrodes. However, this reduction in fidelity was negligible, as detecting epileptiform activity remained adequate.

Forsyth et al. ${ }^{8}$ sought to evaluate the efficacy of an endovascular neural interface with respect to decoding motor activity as a proof-of-concept BMI. Utilizing forced-choice task, 2 sheep were trained to perform left and right gross-motor movements to touch a button within their cage. Sensorimotor signals generated in response to these movements were then recorded and used to classify movement states with a support vector machine (SVM) algorithm. Sheep 1 only contained the Stentrode, whereas sheep 2 contained the Stentrode, subdural, and epidural 

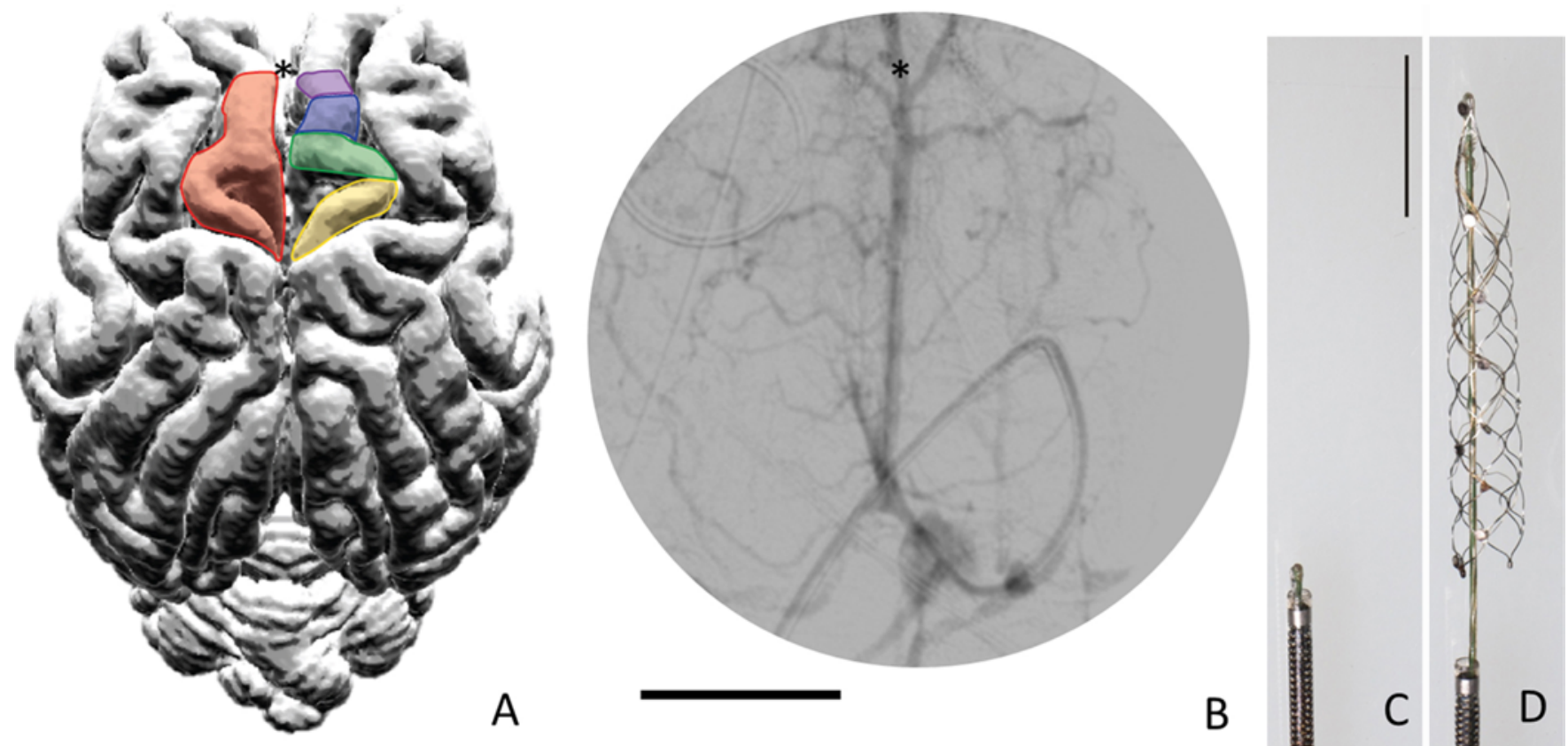

FIG. 2. A: Diagram of a sheep brain showing the motor cortex (red), and somatotopic representations of the hindlimb (yellow), forelimb (green), head and eyes (b/ue), and facial muscles (purple). B: Contrast-enhanced venogram of a sheep highlighting the cortical vessels. The asterisk indicates the desired location of the stent tip in A and B. Bar $=2 \mathrm{~cm}$. C: Stent-electrode array (Stentrode) inside the 1.04-mm internal diameter delivery catheter prior to deployment. Bar $=1 \mathrm{~cm}$. D: Fully expanded Stentrode. Republished with permission of IOP Publishing, Ltd, from Chronic impedance spectroscopy of an endovascular stent-electrode array: Opie NL, John SE, Rind GS, et al., J Neural Eng 13(4): 046020, 2016; permission conveyed through Copyright Clearance Center, Inc.

arrays. Sheep 1 achieved a peak classification accuracy of $70 \%$, while in sheep 2 only a $58 \%$ classification accuracy was observed with the Stentrode. Epidural and subdural accuracies were similar at $51 \%$ and $55 \%$, respectively. The classification accuracies for all electrode types were below chance levels for lateralization of left versus right movement, which the authors attribute to a low number of trials in sheep 2. These findings provide preliminary evidence in support of the use of the Stentrode as a BMI, which is in agreement with the findings of John et al., ${ }^{6}$ who also demonstrated comparable decoding performance among the electrode types.

\section{Intravascular Brain Stimulation}

In a proof-of-concept study, Opie et al..$^{13}$ sought to evaluate the efficacy of the Stentrode with respect to cortical stimulation in 25 sheep (Fig. 2). Stentrodes were placed for at least 4 weeks prior to stimulation to allow for stabilization and electrode integration within the endothelium. A pattern of electrical stimulation was noted along the anterior-posterior axis of the SSS with lip responses, compared to areas with responses of the face, jaw, neck, and limb, occurring at unique locations within the sinus $(\mathrm{p}=0.0433, \mathrm{p}=0.0010, \mathrm{p}<0.0001$, and $\mathrm{p}=0.0004$, respectively). Currents required to elicit a response ranged from approximately 4 to $6 \mathrm{~mA}$. Utilizing angiographic reconstruction (microcomputed tomography), the authors determined electrode orientation did not impart any differences in induced movements; however, increasing orientation from $0^{\circ}($ mean $3.85 \pm 0.85[\mathrm{SEM}] \mathrm{mA}, \mathrm{n}=5)$ to $180^{\circ}(6.85 \pm 0.27 \mathrm{~mA}, \mathrm{n}=5)$ did influence threshold current. No differences were observed when comparing stimulation from the Stentrode to stimulation delivered by penetrating and cortical arrays within the sinus, suggesting a stent-electrode array is a reasonable minimally invasive alternative to invasive recording strategies.

Gerboni et al. ${ }^{14}$ also examined the efficacy of endovascular electrical stimulation in the adult female sheep. The Stentrode was placed in the left transverse sinus and imparted electrical stimulation to evoke neural responses. These cortical evoked potentials were then measured with a subdural electrode grid. The threshold for neural activation appeared to be approximately $2 \mathrm{~mA}$. To determine if these evoked potentials were induced by the Stentrode and not another source, the sheep were visually stimulated in a dark room. The array detected neural activation mostly among the anterior electrodes, indicating the neuronal activity being recorded is distinct in origin from the visual stimulations, which were detected most strongly in the posterior electrodes.

Utilizing computational modeling, Teplitzky et al. ${ }^{15}$ sought to determine potential deep brain stimulation (DBS) targets for an endovascular approach (Fig. 3). From a retrospective MRI analysis, 17 proposed targets for DBS were identified. Using 3D reconstruction, target structures with adjacent veins of sufficient caliber $(>1 \mathrm{~mm})$ were identified. Five targets were identified in proximity to a viable vascular structure, including the ventral aspect of the fornix, anterior nucleus of the thalamus, nucleus accumbens, medial aspect of the subgenual cingulate, and 

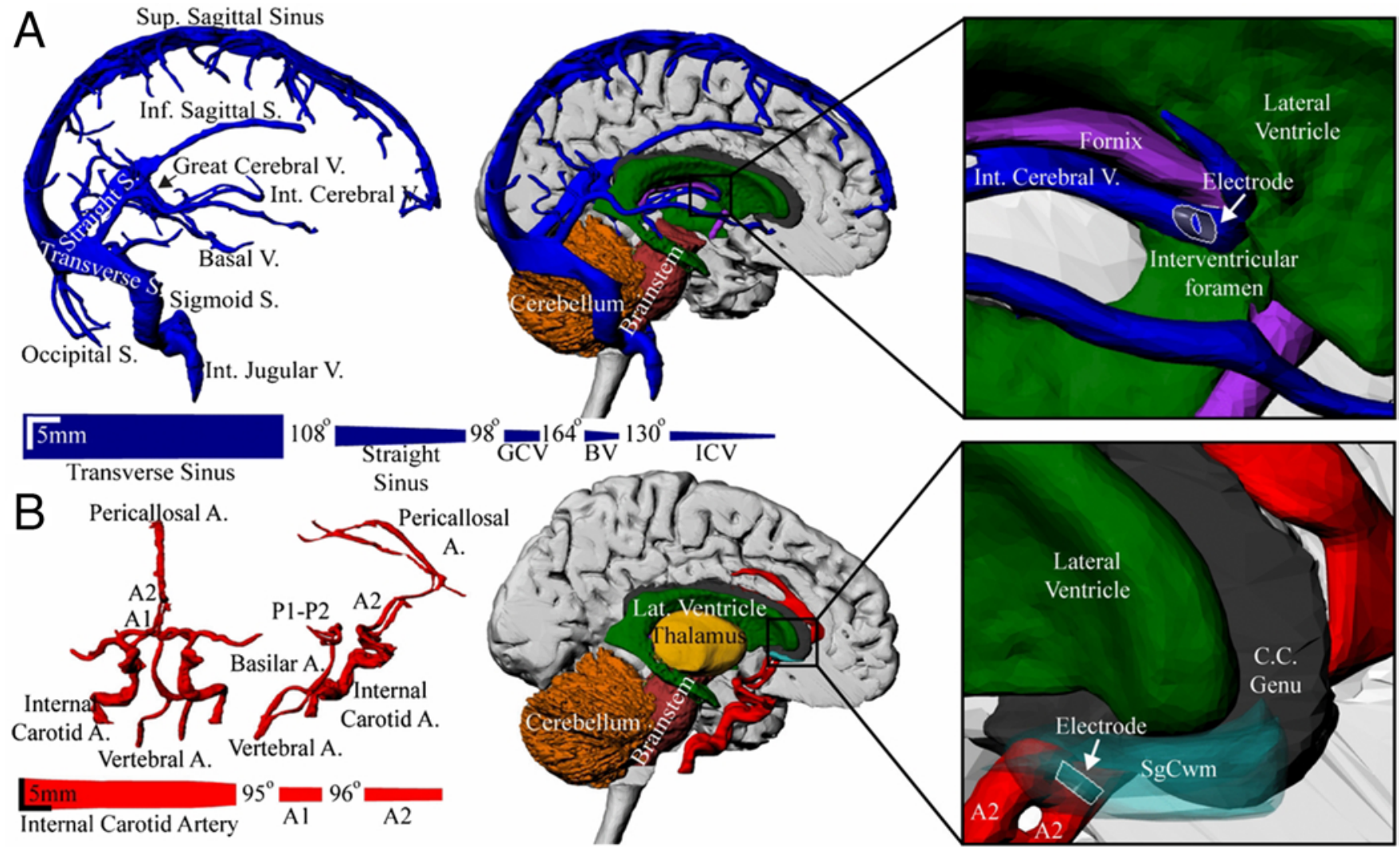

FIG. 3. Endovascular targeting to the fornix (A) and subgenual cingulate white matter ( $\mathrm{SgCwm})(\mathbf{B})$. Vascular reconstructions with estimated path length, vessel diameter, and vessel branching angles (left). Combined vascular and brain reconstructions (center). Ring-electrode model implant location (right). A. = artery; BV = basal vein; C.C. = corpus callosum; GCV = great cerebral vein; ICV = internal cerebral vein; Inf. = inferior; Int. = interior; S. = sinus; Sup. = superior; V. = vein. Republished with permission of IOP Publishing, Ltd, from Computational modeling of an endovascular approach to deep brain stimulation: Teplitzky BA, Connolly AT, Bajwa JA, Johnson MD, J Neural Eng 11(2): 026011, 2014; permission conveyed through Copyright Clearance Center, Inc.

the ventral capsule. Simulations of the theoretical degree of neuronal activation by various designs indicated that a ring-electrode design would likely be more efficient than a guidewire alternative; however, improper placement of the ring would likely result in a significant decrease in potency with respect to neuronal activation. Finally, models were used to compare the activation thresholds of two regions of the brain with respect to endovascular versus traditional stereotactic stimulation. The results showed that activation thresholds were statistically similar between the two modalities, with the added advantage of endovascular techniques appearing to have a greater effect on the contralateral structures as well.

\section{Long-Term Device Placement}

Oxley et al. ${ }^{4}$ published the results of an evaluation of a long-term implantable endovascular device. The devices were implanted into sheep and monitored over the next several weeks to characterize the pattern of impedance over time in the system. Electrodes that were more quickly incorporated into the vessel walls demonstrated improved recording sensitivity. The spatial resolutions and content of the endovascular recordings were similar in quality to an epidural sensor array. The long-term viability of the Stentrode was determined by changes in bandwidth and SSS internal lumen patency from the time of implantation up to 190 days. The maximum bandwidth was found to be stable throughout this period. In vivo SSS lumen diameter measurements up to 12 weeks were performed, followed by ex vivo synchrotron imaging after killing the sheep. No reduction in SSS lumen diameters was observed up to 12 weeks, and patency was confirmed at 190 days. It should be noted that sheep received $100 \mathrm{mg}$ of aspirin daily to minimize thrombosis.

Opie et al. ${ }^{16}$ examined the characteristics of endovascular interfaces over a 6-month period. Electrodes were placed in the SSSs of sheep. Phase angle variability was observed until day 8 , when it then stabilized through day 91. The average maximum bandwidth was consistent across all subjects and time points throughout the 6-month study period. Imaging over the 6-month period demonstrated a small decrease in vessel diameter. At the 100-day mark, none of the images indicated any sign of vascular occlusion, with more than $70 \%$ of struts being covered in neointima, which may represent a decreased risk of thrombosis. Endovascular arrays continue to produce high-quality data with minimal signs of vascular compromise at 6 months.

In another study, Opie et al. ${ }^{17}$ monitored the characteristics of the electrodes in sheep models over a period 
of 190 days $(n=15)$. The electrodes were placed into the SSS overlying the motor cortex. Over the next 91 days, the electrical signals of the electrodes were monitored using electrochemical impedance spectroscopy. At 190 days, the sheep were culled, and histological analysis showed the phase angles stabilized by postoperative day 8 , which is thought to be secondary to changes in the capacitance secondary to changes in the electrode-tissue interface. These findings were supported by the histological analysis, which showed $85 \%$ of struts with neointimal coverage by the 2 -week mark. The findings overall demonstrate that the implants are generally well tolerated over at least a 190-day period.

\section{Optimal Catheter Gauge}

Oxley et al. ${ }^{18}$ sought to formalize a method of cerebral venography to validate an endovascular neural interface. Catheter venography was performed in sheep and several systems were used to determine optimal delivery to the anterior SSS in proximity to the motor cortex. Procedural attempts with the 6-Fr catheters took much more time (median 61 minutes) and were successful $81 \%$ of the time as compared to the 5-Fr system, which had higher rates of complications $(33 \%, 6 / 18)$ and failures $(72 \%)$, but with significantly improved time (median 43 minutes). The best performing system was the 4-Fr catheter, which was successful in all attempts, demonstrated no complications, and had a median completion time of 38 minutes. It is important to note that the 2-Fr catheter had similar results, but the goal of the experiment was to identify the largest-caliber system that optimally accessed the motor area, thus making the 4-Fr catheter preferable.

\section{Microelectrode Material}

Wong et al. ${ }^{19}$ examined the viability of Nitinol-based microelectrodes as electrocorticography tools. Nitinol is of particular interest as previous work has demonstrated that it forms a protective surface in vivo, increasing its stability. Nitinol demonstrated greater magnitude and phase angle than stainless steel and platinum alternatives. Nitinol was able to record changes in neural activity with similar SNR metrics as stainless steel and platinum electrodes. However, Nitinol electrodes demonstrated higher impedance magnitude and a large phase shift for all frequencies. Despite this, these initial results indicate that Nitinol is a promising alternative material as it performs similarly to platinum and stainless steel in many metrics.

Platinum was further explored by Watanabe et al. ${ }^{20}$ This study developed a platinum-based nanowire, demonstrating its feasibility in intravascular recording within the spinal cord of Xenopus laevis. In addition, the study sought to determine the optimal electrical and mechanical properties of the nanowire. Similarly, Llinás et al. ${ }^{21}$ also developed a nanowire, instead using conductive polymer. This polymer was found to have metallic-like conductivity without containing any metallic elements. This is a result of the conjugated structure of the molecular backbone, with alternating single and double bonds. As a result, addition of a charged molecule allows for displacement of weak pi electrons, permitting conduction. The advantage of polymer is that much smaller diameters can be achieved without compromising flexural rigidity and other mechanical properties, enabling superior nanoelectrode implantation into capillaries. ${ }^{20,21}$ The biocompatibility of this conducting polymer nanowire appears to be favorable, although more research is required.

\section{Clinical Studies}

Endovascular neural interfaces have also been demonstrated in human subjects, the first of which occurred in 1973 by Penn et al. ${ }^{7}$ Nearly 2 decades later, Nakase et al. ${ }^{22}$ published their findings in a series of 14 patients undergoing catheterization for treatment of arteriovenous malformation $(n=6)$ or epilepsy $(n=8)$. This study utilized an insulated Seeker Lite-10 guidewire. In all patients, a 2-5 times stronger electroencephalographic voltage potential was found for intracranial recordings when compared to scalp EEG. In addition, subdural electrodes in 3 patients were found to have comparable measurements with intraarterial electrode measurements. Stoeter et al. ${ }^{23}$ utilized intraarterial polytef-insulated Seeker-10 guidewires in 23 patients, comparing all measurements to extracranial electrode readings. The guidewire was placed in the middle meningeal artery and advanced along the frontoparietal branch. They found a positional dependence with respect to somatosensory evoked potential measurement, with no recordings after electrode retraction below the cranial base. Additionally, the readings obtained from the guidewire were found to agree with extracranial registrations. Several other studies also demonstrated the feasibility of endovascular neural recording in humans via the middle cerebral artery, as well as the cavernous sinus, and are summarized in Table $1 .^{24-28}$

\section{Discussion}

In this systematic review, the authors summarize the current state of the field with respect to endovascular neural interfaces. By utilizing the cerebrovascular system as a natural conduit to the brain, a minimally invasive BMI is achievable. Our findings suggest that endovascular measurements of neural activity are feasible, but further validation and refinement is necessary before Stentrodes may serve as a viable adjuvant or even alternative to traditional approaches in the future. $5,6,8,12,13$

A decrease in spatial resolution and signal detection is observed in predominantly lower-frequency ranges $(<$ $30 \mathrm{~Hz}$ ) with intravascular electrode placement. ${ }^{5,12}$ This is thought to be the result of greater resistance imparted by the blood vessel-electrode interface at this frequency range. ${ }^{12}$ Despite this, these findings are believed to be clinically negligible given adequate detection of epileptiform activity and measurements across a broad range of frequencies. ${ }^{5,12}$

With respect to motor classification, only a single study was identified assessing signal decoding. ${ }^{8}$ In sheep, the authors demonstrate the feasibility of Stentrode decoding in a 2-class (right vs left limb) decoding paradigm, achieving a peak classification of $70 \%$ with SVM. While promising, it should be noted that accuracies much higher have been shown in humans for 2-class decoding paradigms. ${ }^{29}$ Subdural electrode decoding is typically greater than $95 \%$ in 
humans, with scalp EEG right-left motor decoding greater than $85 \%$ being achievable. ${ }^{29-32}$ Given variations in classification schemes and optimization algorithms, in addition to a lack of human motor classification studies utilizing Stentrodes, further validation of Stentrode decoding is necessary before direct comparisons can be made.

The effectiveness of intravascular brain stimulation with Stentrodes was also explored, with either ovine experiments or computational modeling. ${ }^{13-15}$ The threshold for neuronal activation was between $2 \mathrm{~mA}$ (left transverse sinus) and 4-6 mA (SSS), appearing to be location dependent. Unique neural responses were able to be elicited depending on the site of cortical stimulation. ${ }^{13,14}$ Teplitzky et al. ${ }^{15}$ attempted to model DBS targets based on proximity to viable vascular structures. By utilizing 3D reconstruction, the authors identified various deep brain targets given adjacent vessels of viable caliber. In addition, activation thresholds were calculated to be similar to stereotactic stimulation. While this model does not fully emulate neuronal mechanics and morphology, it opens the realm for future experimental studies exploring endovascular DBS. In general, the concept of minimally invasive endovascular brain stimulation remains novel, and further exploration with respect to bipolar versus monopolar stimulation, pulse duration, and degree of neural spread is warranted. ${ }^{13-15}$

Given that both the long-term viability and risk of venous thrombosis development are unclear, the long-term (190-day) implantation of venous electrodes was explored in 3 studies., ${ }^{4,16,17}$ Stent incorporation, which relies on neointimal proliferation and endothelialization, was shown to occur between 6 and 8 days given a significant reduction in phase angle variability at this time point. All 3 studies demonstrated consistent production of data with minimal signs of vascular compromise at 6 months. While angiography revealed vessel lumen diameters to decrease over time, venous patency was histologically demonstrated at 190 days., ${ }^{4,16,17}$ Only Oxley et al. provided sheep with 100 $\mathrm{mg}$ of aspirin daily to minimize thrombosis. ${ }^{4}$ With respect to maximum bandwidth, epidural and vascular arrays performed similarly $(200 \pm 6 \mathrm{~Hz}, 1$-way ANOVA, $\mathrm{p}=0.66)$, although Stentrode performance was significantly reduced when compared to subdural arrays $(27 \pm 7 \mathrm{~Hz}, 1$-way ANOVA, $\mathrm{p}<0.001) .{ }^{4}$ These findings suggest long-term Stentrode implantation is viable, although further verification and fine-tuning is required with respect to minimizing stroke and thrombus risk (antiplatelets, stent coatings, etc.), reducing mechanical failure rates (wire fatigue associated with repeated neck movement), and maximizing electrode sensitivity. $4,10,16,17$

Other important factors to consider are the catheter gauge and material type. Based on a single study by Oxley et al.,18 the 4-Fr catheter performed best when compared to 6-Fr and 5-Fr catheters. Nitinol, platinum, and polymerbased microelectrodes have all been explored, with Nitinol appearing superior to platinum; however, conductive polymer nanowire confers the advantage of markedly reduced diameters while maintaining flexural rigidity and metallic-like conductivity. ${ }^{19-21}$ Further studies comparing electrode characteristics against traditional subdural and epidural arrays are necessary before determining ideal electrode gauge and material.
Clinical studies remain limited, although earlier reports demonstrate that accurate and consistent measurement of evoked potentials is feasible intravascularly, with more recent studies utilizing venous approaches. . $^{5,6,8,12,13}$ Earlier human studies show that intraarterial measurements are also feasible; however, these studies were largely performed secondary to primary endovascular intervention., ${ }^{722-28}$ Currently, the Stentrode First in Human Early Feasibility Study (SWITCH, https://clinicaltrials.gov no.: NCT03834857) is in the active recruitment phase, with the primary outcome being treatment-associated adverse outcomes.

There are several limitations to the reviewed data that should be noted. No standard protocol exists for endovascular Stentrode material, gauge, placement, or decoding classification algorithms. This introduces a large element of heterogeneity that should be considered when drawing comparisons. Additionally, studies varied greatly in experimental design, and an overall low number of studies for each category discussed further hinders interpretability. Given the nascency of an endovascular BMI, the majority of data rely on animal or computation models, with the last human study being performed 20 years ago.

\section{Conclusions}

The utility of endovascular electrode placement lies in its noninvasive nature and ability to use the vasculature as a conduit for measuring both cortical and deep brain activity. Endovascular electrode signal detection appears to be feasible after short- and long-term implantation, and preliminary data suggest measurements similar to traditional approaches; however, these findings were largely inconsistent and require further validation. Adequate brain stimulation is also achievable. Further study is required with respect to both decoding and safety in human trials before a formal BMI can be developed with endovascular electrodes.

\section{References}

1. Hochberg LR, Bacher D, Jarosiewicz B, et al. Reach and grasp by people with tetraplegia using a neurally controlled robotic arm. Nature. 2012;485(7398):372-375.

2. Hamer HM, Morris HH, Mascha EJ, et al. Complications of invasive video-EEG monitoring with subdural grid electrodes. Neurology. 2002;58(1):97-103.

3. Saxena T, Karumbaiah L, Gaupp EA, et al. The impact of chronic blood-brain barrier breach on intracortical electrode function. Biomaterials. 2013;34(20):4703-4713.

4. Oxley TJ, Opie NL, John SE, et al. Minimally invasive endovascular stent-electrode array for high-fidelity, chronic recordings of cortical neural activity. Nat Biotechnol. 2016;34(3):320-327.

5. Bower MR, Stead M, Van Gompel JJ, et al. Intravenous recording of intracranial, broadband EEG. J Neurosci Methods. 2013;214(1):21-26.

6. John SE, Opie NL, Wong YT, et al. Signal quality of simultaneously recorded endovascular, subdural and epidural signals are comparable. Sci Rep. 2018;8(1):8427.

7. Penn RD, Hilal SK, Michelsen WJ, et al. Intravascular intracranial EEG recording. Technical note. J Neurosurg. 1973;38(2):239-243.

8. Forsyth IA, Dunston M, Lombardi G, et al. Evaluation of a minimally invasive endovascular neural interface for decod- 
ing motor activity. In: 2019 9th International IEEE/EMBS Conference on Neural Engineering (NER). IEEE; 2019:750753.

9. John SE, Grayden DB, Yanagisawa T. The future potential of the Stentrode. Expert Rev Med Devices. 2019;16(10):841-843.

10. Rajah G, Saber H, Singh R, Rangel-Castilla L. Endovascular delivery of leads and Stentrodes and their applications to deep brain stimulation and neuromodulation: a review. $\mathrm{Neu}$ rosurg Focus. 2018;45(2):E19.

11. Sefcik RK, Opie NL, John SE, et al. The evolution of endovascular electroencephalography: historical perspective and future applications. Neurosurg Focus. 2016;40(5):E7.

12. John SE, Apollo NV, Opie NL, et al. In vivo impedance characterization of cortical recording electrodes shows dependence on electrode location and size. IEEE Trans Biomed Eng. 2019;66(3):675-681.

13. Opie NL, John SE, Rind GS, et al. Focal stimulation of the sheep motor cortex with a chronically implanted minimally invasive electrode array mounted on an endovascular stent. Nat Biomed Eng. 2018;2(12):907-914.

14. Gerboni G, John SE, Ronayne SM, et al. Cortical brain stimulation with endovascular electrodes. Conf Proc IEEE Eng Med Biol Soc. 2018;2018:3088-3091.

15. Teplitzky BA, Connolly AT, Bajwa JA, Johnson MD. Computational modeling of an endovascular approach to deep brain stimulation. J Neural Eng. 2014;11(2):026011.

16. Opie NL, Rind GS, John SE, et al. Feasibility of a chronic, minimally invasive endovascular neural interface. Conf Proc IEEE Eng Med Biol Soc. 2016;2016:4455-4458.

17. Opie NL, John SE, Rind GS, et al. Chronic impedance spectroscopy of an endovascular stent-electrode array. J Neural Eng. 2016;13(4):046020.

18. Oxley TJ, Opie NL, Rind GS, et al. An ovine model of cerebral catheter venography for implantation of an endovascular neural interface. J Neurosurg. 2018;128(4):1020-1027.

19. Wong YT, Opie NL, John SE, et al. Suitability of Nitinol electrodes in neural prostheses such as endovascular neural interfaces. Conf Proc IEEE Eng Med Biol Soc. 2016;2016:4463-4466.

20. Watanabe H, Takahashi $\mathrm{H}$, Nakao M, et al. Intravascular neural interface with nanowire electrode. Electron Commun Jpn. 2009:92(7):29-37.

21. Llinás RR, Walton KD, Nakao M, et al. Neuro-vascular central nervous recording/stimulating system: using nanotechnology probes. J Nanopart Res. 2005;7(2):111-127.

22. Nakase H, Ohnishi H, Touho H, et al. An intra-arterial electrode for intracranial electro-encephalogram recordings. Acta Neurochir (Wien). 1995;136(1-2):103-105.

23. Stoeter P, Dieterle L, Meyer A, Prey N. Intracranial electroencephalographic and evoked-potential recording from intravascular guide wires. AJNR Am J Neuroradiol. 1995;16(6):1214-1217.

24. Boniface SJ, Antoun N. Endovascular electroencephalography: the technique and its application during carotid amytal assessment. J Neurol Neurosurg Psychiatry. 1997;62(2):193195.
25. García-Asensio S, Guelbenzu S, Barrena R, Valero P. Technical aspects of intra-arterial electroencephalogram recording. Interv Neuroradiol. 1999;5(4):289-300.

26. Kunieda T, Ikeda A, Mikuni N, et al. Use of cavernous sinus EEG in the detection of seizure onset and spread in mesial temporal lobe epilepsy. Epilepsia. 2000;41(11):1411-1419.

27. Mikuni N, Ikeda A, Murao K, et al. "Cavernous sinus EEG": a new method for the preoperative evaluation of temporal lobe epilepsy. Epilepsia. 1997;38(4):472-482.

28. Thömke F, Stoeter P, Stader D. Endovascular electroencephalography during an intracarotid amobarbital test with simultaneous recordings from 16 electrodes. J Neurol Neurosurg Psychiatry. 1998;64(4):565.

29. Leuthardt EC, Schalk G, Wolpaw JR, et al. A brain-computer interface using electrocorticographic signals in humans. $J$ Neural Eng. 2004;1(2):63-71.

30. Fujiwara Y, Matsumoto R, Nakae T, et al. Neural pattern similarity between contra- and ipsilateral movements in highfrequency band of human electrocorticograms. Neuroimage. 2017;147:302-313.

31. Scherer R, Zanos SP, Miller KJ, et al. Classification of contralateral and ipsilateral finger movements for electrocorticographic brain-computer interfaces. Neurosurg Focus. 2009;27(1):E12.

32. Wang Y, Hong B, Gao X, Gao S. Design of electrode layout for motor imagery based brain-computer interface. Electron Lett. 2007:43(10):557-558.

\section{Disclosures}

The authors report no conflict of interest concerning the materials or methods used in this study or the findings specified in this paper.

\section{Author Contributions}

Conception and design: Syed, Soldozy. Acquisition of data: Soldozy, Young. Analysis and interpretation of data: Soldozy, Young. Drafting the article: Soldozy. Critically revising the article: Syed, Soldozy, Kumar, Capek. Reviewed submitted version of manuscript: all authors. Approved the final version of the manuscript on behalf of all authors: Syed. Administrative/technical/ material support: Syed, Felbaum, Jean, Park. Study supervision: Syed, Felbaum, Jean, Park.

\section{Correspondence}

Hasan R. Syed: University of Virginia Health System, Charlottesville,VA. syedhr@gmail.com. 